\title{
Risk Analysis and Evaluation of Wind Electric Farm Construction
}

\author{
Gang Chen \\ School of Information Management and Engineering, \\ Shanghai University of Finance and Economics, \\ Shanghai, China
}

\begin{abstract}
Wind energy resources are rich in China. At the end of 2012, China's wind power installed capacity has reached 75.324 GW, at the top list in the world. However in China, high-speed development of wind power industry has brought great risks. In this paper, after decomposition of identified four aspects of risk factors, there is also a risk evaluation index system in paper. According to the risk characteristics of wind electric farm and fuzziness of human thinking, utilizing the advantages of triangular fuzzy number and fuzzy complementary judgment matrix and being combined with fuzzy comprehensive evaluation. In paper there is a model based on fuzzy triangular numbers for risk analysis and evaluation. Based on the evaluation results, there are some feasible risk disposal advices.
\end{abstract}

Keywords-wind electric farm; triangular fuzzy number; risk analysis; evaluation

\section{INTRODUCTION}

In China, construction of Wind electric farm developed very quickly. At the end of 1989, it is just 4200 $\mathrm{kW}$ but in 2010 it is up to 75,340,000 kW, ranking first in the world. ${ }^{[1]}$ But from 2010, with the economy going down, electricity demand continued to decline. ${ }^{[2]}$ Wind electric farm's profit is declining. Wind electric farm has become a high cost industry. Construction and investment risk of Wind electric farm is increasing.

\section{RISK IDENTIFICATION OF WIND ELECTRIC FARM CONSTRUCTION}

The key to reducing the risk of wind field construction is risk control in three stages such as planning, construction, and production.

\section{A. Process of Wind Electric Farm Construction Risk Identification}

First of all, using the SWOT analysis threat, opportunity, advantages that wind electric farm project facing and made qualitative analysis on the internal and external environment of wind electric farm project have ${ }^{[3]}$. We can sum up the potential risks of wind electric field. According to the internal rate of return IRR of wind electric farm, we can identify sensitivity analysis from a direct impact on the profitability index risk project. The profit levels determine the degree of influence of risk. Then we can get risk list as based on risk analysis and assessment ${ }^{[4]}$.

Technological process of wind electric farm construction risk analysis and assessment just as Figure 1.

According to Table I, risk assessment of wind electric farm construction is just as following. 


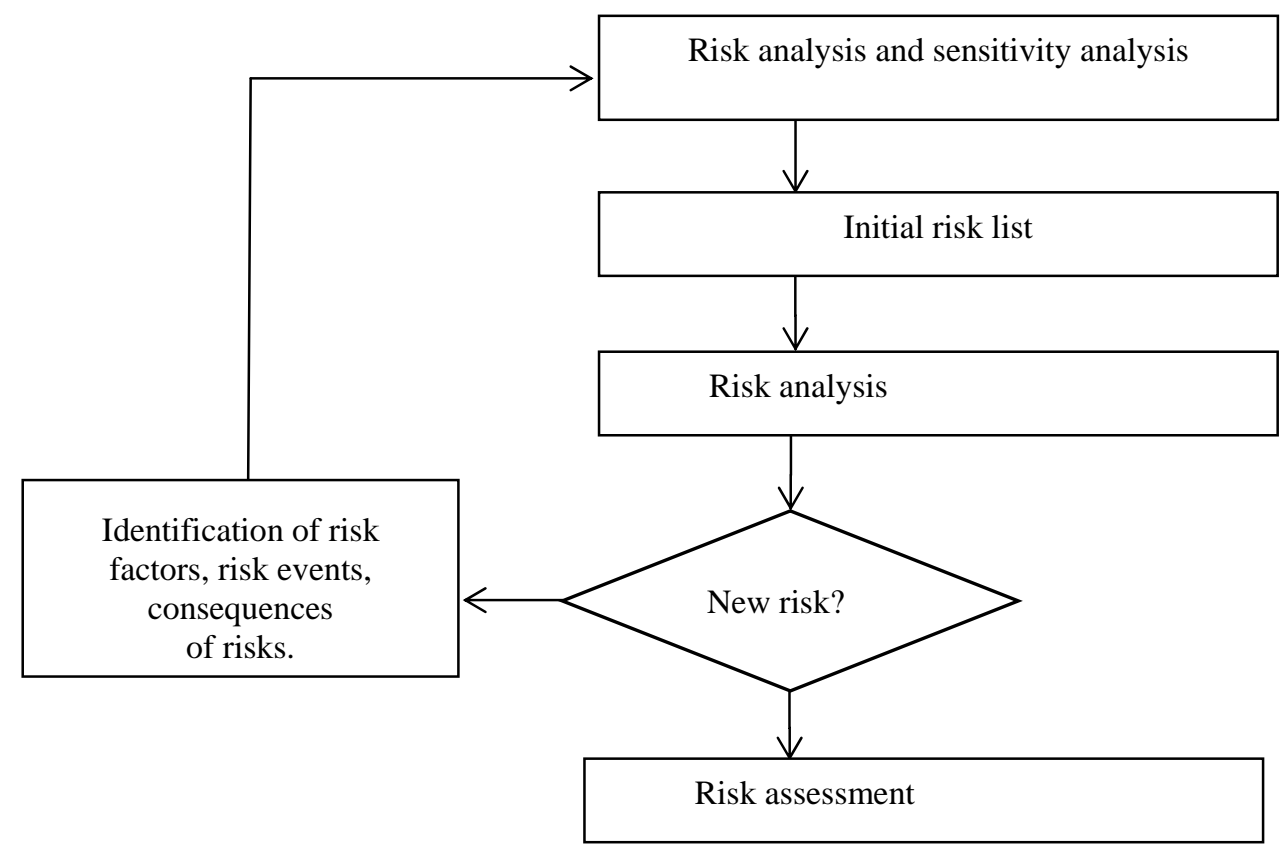

Figure 1. Technological process of wind electric farm construction risk analysis and assessment.

TABLE I. RISK ASSESSMENT OF WIND ELECTRIC FARM CONSTRUCTION.

\begin{tabular}{|c|c|c|c|}
\hline \multirow{13}{*}{ External Risk } & Fist Level & Second Level & Risk Scenario Description \\
\hline & \multirow{4}{*}{$\begin{array}{l}\text { Political and economic risk } \\
U_{1}\end{array}$} & Government support $U_{11}$ & State subsidies \\
\hline & & Price policy $U_{12}$ & State price \\
\hline & & $\begin{array}{l}\text { Laws and } \\
\text { regulations } U_{13}\end{array}$ & Legal adjustment \\
\hline & & Financing rate $U_{14}$ & Loan program \\
\hline & \multirow[t]{4}{*}{ Social and legal risk $U_{4}$} & Public Security $U_{21}$ & Damaged equipment \\
\hline & & Climate $U_{22}$ & Influence of extreme temperature for air density \\
\hline & & Natural disaster $U_{23}$ & Earthquake, fire, flood, lightning \\
\hline & & $\begin{array}{l}\text { Geological } \\
\text { conditions } U_{24}\end{array}$ & Improper location \\
\hline & \multirow{4}{*}{$\begin{array}{l}\text { Technical and } \\
\text { management } U_{3}\end{array}$} & Waste electrical $U_{31}$ & Too much electricity \\
\hline & & Construction organization $U_{32}$ & $\begin{array}{l}\text { Construction schedule delay, Construction equipment is not complete, } \\
\text { The construction quality is not reliable }\end{array}$ \\
\hline & & Operation and maintenance $U_{33}$ & $\begin{array}{l}\text { Repair and maintenance is not seasonable, Lack of repair materials } \\
\text { and equipment, Inadequate safety regulations }\end{array}$ \\
\hline & & Wind energy resources $U_{34}$ & $\begin{array}{l}\text { Wind data is not accurate, The location is not correct, The actual use } \\
\text { of time is low }\end{array}$ \\
\hline \multirow{4}{*}{\multicolumn{2}{|c|}{ Internal risk $U_{4}$}} & Development obstacles $U_{41}$ & Not through the registration \\
\hline & & Fan selection $U_{42}$ & $\begin{array}{l}\text { Single machine reliability is lower than } 98 \% \text {, The reliability of less } \\
\text { than } 95 \%\end{array}$ \\
\hline & & $\begin{array}{l}\text { Contract } \\
\text { management } U_{43}\end{array}$ & $\begin{array}{l}\text { Contract terms omission, The terms of the contract the expression is } \\
\text { wrong, The claim is ineffective, Contract disputes }\end{array}$ \\
\hline & & Project team $U_{44}$ & $\begin{array}{l}\text { Project management mode selection is improper, low quality of } \\
\text { personnel, Occupation moral hazard }\end{array}$ \\
\hline
\end{tabular}


B. Process of Wind Electric Farm Construction Risk Analysis and Assessment

1) Set Risk List

According to wind electric farm construction risk analysis and assessment, risk index element set will be divided into two stages ${ }^{[5]}$.

a) First Level of Risk Index Element Set $P=\left\{U_{1}, U_{2}, \ldots, U_{i}\right\}$

b) Second Level of Risk Index Element Set

$$
\begin{gathered}
u_{1}=\left\{u_{11}, u_{12}, \ldots, u_{1 j}\right\} \\
u_{2}=\left\{u_{21}, u_{22}, \ldots, u_{2 j}\right\} \\
\ldots ., \quad i=4, j=4 \\
u_{i}=\left\{u_{i 1}, u_{i 2}, \ldots, u_{i j}\right\} \quad
\end{gathered}
$$

c) Establishment of Risk Evaluation Set

According to influence of risks to wind electric farm construction, we can get evaluation sets:

$$
\mathrm{V}=\left\{v_{1}, v_{2}, v_{3}, v_{4}, v_{5}\right\}
$$

$=\{$ very high risk, high risk, medium risk, low risk, very

$$
\text { low risk\} }
$$

2) Set fuzzy Evaluation Matrix of Single Factor

There are two characteristics of risks-Probability of occurrence $P$ and loss of risk $L$. Using these two features as target to evaluate all risk elements we can get fuzzy relation $R_{L}$ and $R_{P}$ of risk factors set $U_{1}$ and Risk evaluation set $V$ according to the condition of $P$ and $L$ risk factors set. We find five Experts. They gave evaluation value.

$$
\begin{aligned}
R_{L} & =\left[\begin{array}{llll}
r_{L 11} & r_{L 12} & \ldots & r_{L 15} \\
r_{l 21} & r_{l 22} & \ldots & r_{L 25} \\
r_{L m 1} & r_{L m 2} & \ldots & r_{L m 5}
\end{array}\right] \\
R_{p} & =\left[\begin{array}{llll}
r_{p 11} & r_{p 12} & \ldots & r_{p 15} \\
r_{p 21} & r_{p 22} & \ldots & r_{p 25} \\
r_{p m 1} & r_{p m 2} & \ldots & r_{p m 5}
\end{array}\right]
\end{aligned}
$$

Suppose $m$ is the number of two level index elements. No prior relationship exists between $P$ and $L$. According to the parallel relationship between $R_{L}$ and $R_{P}$ we can put $R_{L}$ and $R_{P}$ into a fuzzy single factor risk judgment matrix

$R=\left[\begin{array}{lll}r_{11} & r_{12} & \ldots r_{15} \\ r_{21} & r_{22} & \ldots r_{25} \\ r_{m 1} & r_{m 2} & \ldots r_{m 5}\end{array}\right]$

\begin{tabular}{|c|c|c|c|c|}
\hline $\begin{array}{l}\text { Standards of } \\
U_{i}\end{array}$ & $u_{i 1}$ & $u_{i 2}$ & . . & $u_{i j}$ \\
\hline$u_{i 1}$ & \multirow{4}{*}{\multicolumn{4}{|c|}{$\begin{array}{l}\text { The expert scaling with triangular fuzzy numbers } \\
\text { according to Table } 3.1 \text {.as }\left(l_{i j}, m_{i j}, s_{i j}\right) . l_{i j} \text { is the most } \\
\text { pessimistic value. } m_{i j} \text { is the most probable value. } s_{i j} \\
\text { is the most optimistic value. } \\
l_{i i}=m_{i i}=s_{i i}=0.5, l_{i j}+s_{j i}=m_{i j}+m_{j i}=s_{i j}+I_{j i}=1\end{array}$}} \\
\hline$u_{i 2}$ & & & & \\
\hline$\ldots$ & & & & \\
\hline$u_{i j}$ & & & & \\
\hline
\end{tabular}

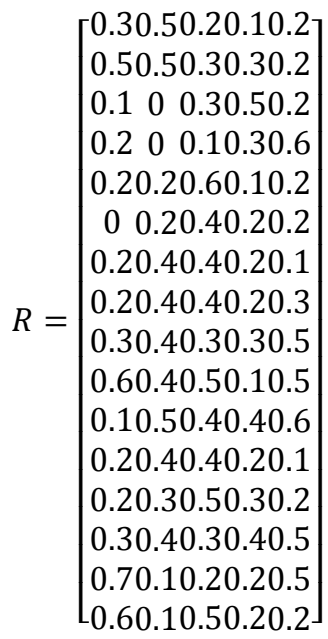

TABLE II. 0.1 TO 0.9 SCALE.

\begin{tabular}{|l|l|}
\hline Scale & The Meaning of Two Elements Compared to \\
\hline 0.5 & Two elements are equally important \\
\hline 0.6 & $\begin{array}{l}\text { The former was slightly more important than the } \\
\text { latter }\end{array}$ \\
\hline 0.7 & The former is important than the latter \\
\hline 0.8 & The former is more important than the latter \\
\hline 0.9 & The former is very important than the latter \\
\hline $0.1,0.2,0.3$, & $\begin{array}{l}\text { If the importance of } U_{i} \text { and } U_{j} \text { is } a_{i j}, U_{j} \text { and } U \\
\text { ratio is } a_{j i}=1-a_{i j}\end{array}$ \\
\hline
\end{tabular}

TABLE III. AN IMPORTANT ELEMENT OF RISK EVALUATION TABLE $(i=4, j=4)$.

According to F-AHP, we should Structure two stage judgment matrix and the consistency check.

$K$ is the number of experts who marks elements of $U_{i}$. According to standards in Table II, such as the importance of quantitative comparison of two scoring, expert can mark elements of $U_{i}$ as Table III.

Construction of $k \mathrm{X} n$ two level index layers we can get comparison judgment matrixes.

$$
\begin{aligned}
& B_{i}^{(q)}=\left(b_{i j}^{(q)}\right)_{n X n}=\left[\begin{array}{lll}
b_{11}^{(q)} b_{12}^{(q)} & \ldots & b_{1 j}^{(q)} \\
b_{21}^{(q)} b_{22}^{(q)} & \ldots & b_{2 j}^{(q)} \\
b_{i 1}^{(q)} b_{i 2}^{(q)} & \ldots & b_{i j}^{(q)}
\end{array}\right] \\
& \qquad(q=1,2, \ldots, k) q \text { is the number of experts? } \\
& \text { value of } b_{i j}^{(q)} \text { is based on Table 2. } \\
& \text { 3) Consistency Check of } B_{i}^{(q)} \text { as Following: } \\
& \quad \text { Fuzzy probability of }\left(l_{i j}, m_{i j}, s_{i j}\right) \text { is } \\
& P_{(l)}=\frac{1}{2(1+N)}
\end{aligned}
$$


$P_{(m)}=\frac{N+2 N M+M}{2(1+N)(1+M)}$

$P_{(s)}=\frac{1}{2(1+M)}$

We can assuming $\left(l_{i j}, m_{i j}, s_{i j}\right)$ has the same probability of occurrence, such as $M=N=1$.

So triangular fuzzy number expected value is $e_{i j}^{(q)}=P_{(l)} \times l_{i j}^{q}+P_{(m)} \times m_{i j}^{q}+P_{(s)} \times s_{i j}^{q}$

Expected value judgment matrix:

$E_{i}^{(q)}=\left(e_{i j}^{(q)}\right)_{n X n}=\left[\begin{array}{ccc}e_{11}^{(q)} e_{12}^{(q)} & \ldots & e_{1 j}^{(q)} \\ e_{21}^{(q)} e_{22}^{(q)} & \ldots & e_{2 j}^{(q)} \\ e_{i 1}^{(q)} e_{i 2}^{(q)} & \ldots & e_{i j}^{(q)}\end{array}\right]$

$(q=1,2, \ldots, k) \quad q$ is the number of experts?

To calculate the weight vector $w_{i}^{(q)}$

$w_{i}=\frac{1}{n}-\frac{1}{2 a}+\frac{1}{a n} \sum_{k=1}^{n} b_{i k}$

$$
i \in N, a \geq(n-1) / 2
$$

Construction of characteristic matrix

$C_{i}^{(q)}=\left(c_{i j}^{(q)}\right)_{n \times n}=\left[\begin{array}{ccc}c_{11}^{(q)} c_{12}^{(q)} & \ldots & c_{1 j}^{(q)} \\ c_{21}^{(q)} c_{22}^{(q)} & \ldots & c_{2 j}^{(q)} \\ c_{i 1}^{(q)} c_{i 2}^{(q)} & \ldots & c_{i j}^{(q)}\end{array}\right]$

$C_{i j}^{(q)}=\mathrm{a}\left(w_{i}^{(q)}-w_{j}^{(q)}\right)+0.5$

$$
(q=1,2, \ldots, k) \quad q \text { is the number of experts? }
$$

Calculated for each comparison, we can get judgment matrix compatibility index $\mathrm{I}\left(B_{i}^{(q)}, C_{i}^{(q)}\right)$. If I $\left(B_{i}^{(q)}, C_{i}^{(q)}\right)<0.1$, then we can get the second level comparison matrix from exporter $q$ is consistency. If $\mathrm{I}\left(B_{i}^{(q)}, C_{i}^{(q)}\right) \geq 0.1$, then second level comparison matrix should be charged.

If second level comparison matrixes $B_{i}^{(q)}$ from $K$ experts are consistent with each other, we can get second level index layer fuzzy comprehensive evaluation matrix $B_{i}$.

$$
\begin{gathered}
B_{j}=\left(b_{i j}\right)_{n \times n}=\left[\begin{array}{lll}
b_{11} b_{12} & \ldots & b_{1 j} \\
b_{21} b_{22} & \ldots & b_{2 j} \\
b_{i 1} b_{i 1} & \ldots & b_{i j}
\end{array}\right] \\
b_{i j}=\left(b_{i j}^{(1)} \oplus b_{i j}^{(2)} \oplus b_{i j}^{(3)} \oplus \ldots . \oplus b_{i j}^{(k)}\right) / k
\end{gathered}
$$

According to Eq. (12), we can get weight vector of second level index layer:

$$
W_{i i}=\left(w^{i 1}, w^{i 2}, \ldots ., w^{i j}\right)^{T}
$$

So

$$
\begin{gathered}
W_{11}=(0.38,0.22,0.92,0.11)^{T} \\
W_{22}=(0.27,0.43,0.25,0.222)^{T} \\
W_{33}=(0.36,0.27,0.22,0.65)^{T} \\
W_{44}=(0.61,0.31,0.24,0.68)^{T}
\end{gathered}
$$

Using the same method, we can get weight vector of first level index:

$W_{L P}=\left(w_{1}^{L P}, w_{2}^{L P}, \ldots, w_{n}^{L P}\right)$

$P$ is probability of risk. $L$ is the risk consequence. Assume that $P$ and $L$ are parallel.

$$
W_{L P}=(0.28,0.58,0.27,0.39)
$$

Assume that there are $\mathrm{m}$ two level index. The element weight vector of first and second level indicators can be synthesis for the total weight vector.

$$
\begin{aligned}
& w=W_{L P} \cdot\left[\begin{array}{lll}
W_{11} & & \\
& \ddots & \\
& & W_{n n}
\end{array}\right]=\left(w_{1}, w_{2}, \ldots, w_{m}\right)^{T} \\
& w=(0.08,0.05,0.07,0.04,0.07,0.06,0.07,0.06 \text {, } \\
& 0.08,0.06,0.06,0.07,0.05,0.07,0.05,0.07)^{T}
\end{aligned}
$$

4) Synthetic Evaluation Results

We get fuzzy evaluation set from synthetic $W$ and $R$ with fuzzy operators

$B=w \times R=\left[\begin{array}{l}0.08 \\ 0.05 \\ 0.07 \\ 0.04 \\ 0.07 \\ 0.06 \\ 0.07 \\ 0.06 \\ 0.08 \\ 0.06 \\ 0.06 \\ 0.07 \\ 0.05 \\ 0.07 \\ 0.05 \\ 0.07\end{array}\right] \times\left[\begin{array}{ll}0.30 .50 .20 .10 .2 \\ 0.50 .50 .30 .30 .2 \\ 0.100 .30 .50 .2 \\ 0.200 .10 .30 .6 \\ 0.20 .20 .60 .10 .2 \\ 00.20 .40 .20 .2 \\ 0.20 .40 .40 .20 .1 \\ 0.20 .40 .40 .20 .3 \\ 0.30 .40 .30 .30 .5 \\ 0.60 .40 .50 .10 .5 \\ 0.10 .50 .40 .40 .6 \\ 0.20 .40 .40 .20 .1 \\ 0.20 .30 .50 .30 .2 \\ 0.30 .40 .30 .40 .5 \\ 0.70 .10 .20 .20 .5 \\ 0.60 .10 .50 .20 .2\end{array}\right]$
$=(0.15,0.34,0.36,0.091$ and 0.089$)$

The maximum value is 0.36 . It seems the project has Medium risk.

\section{RISK MANAGEMENT PLAN}

\section{Risk Factors}

According to the weight sequence we can get second level of risk factors. 
TABLe IV. Weight SEQUenCE OF SECOND LEVEL Risk.

\begin{tabular}{|l|l|}
\hline Second level of risk factors & Sequence \\
\hline Waste electrical $U_{31}$ & 1 \\
\hline Government support $U_{11}$ & 2 \\
\hline Wind energy resources $U_{34}$ & 3 \\
\hline Public Security $U_{21}$ & 4 \\
\hline Fan selection $U_{42}$ & 5 \\
\hline Laws and regulations $U_{13}$ & 6 \\
\hline Natural disaster $U_{23}$ & 7 \\
\hline Climate $U_{22}$ & 8 \\
\hline Operation and maintenance $U_{33}$ & 9 \\
\hline Project team $U_{44}$ & 10 \\
\hline Geological conditions $U_{24}$ & 11 \\
\hline Construction organization $U_{32}$ & 12 \\
\hline Development obstacles $U_{41}$ & 13 \\
\hline Price policy $U_{12}$ & 14 \\
\hline Contract management $U_{43}$ & 15 \\
\hline Financing rate $U_{14}$ & 16 \\
\hline
\end{tabular}

\section{Risk Management Plan}

Risk retention, risk mitigation, risk transfer, risk aversion is commonly used risk management plan.

Top 5 in Table 4 such as Waste electrical $U_{31}$, Government support $U_{11}$. Wind energy resources $U_{34}$, Public Security $U_{21}$, Fan selection $U_{42}$ should be treated as risk aversion and risk mitigation.

Bottom 5 in Table 4 such as Construction organization $U_{32}$, Development obstacles $U_{41}$, Price policy $U_{12}$, Contract management $U_{43}$, financing rate $U_{14}$ could be treated as risk retention.

\section{CONCLUSIONS}

According to the risk characteristics of wind electric farm, utilizing the advantages of triangular fuzzy number and fuzzy complementary judgment matrix and being combined with fuzzy comprehensive evaluation. There is an F-AHP model based on fuzzy triangular numbers for risk analysis and evaluation. Risk retention, risk mitigation, risk transfer, risk aversion is commonly used risk management plan. To the case in paper, Waste electrical $U_{31}$, Government support $U_{11}$. Wind energy resources $U_{34}$, Public Security $U_{21}$, Fan selection $U_{42}$ should be treated as risk aversion and risk mitigation. Construction organization $U_{32}$, Development obstacles $U_{41}$, Price policy $U_{12}$, Contract management $U_{43}$, financing rate $U_{14}$ could be treated as risk retention.

\section{REFERENCES}

[1] Chen Gang, Data center management plan in cloud computing environment. In: 3rd International Conference on Information Management, Innovation Management and Industrial Engineering, ICIII, vol. 4, pp. 393-396, 2010.

[2] Chen Gang, Risk evaluation of business continuity management by using green technology. Communications in Computer and Information Science, 113, pp. 86-92, 2010.

[3] T.L. Satty, Decision making with the analysis hierarchy process, Int. J. Services Science, 1(1), pp. 83-98, 2008.

[4] Chen Gang, Decision-making model of business continuity management. Lecture Notes in Electrical Engineering, 140, pp. 285-289, 2012.

[5] Jennifer Morris, Sergey Paltsev, John Reilly, Marginal Abatement Costs and Marginal Welfare Costs for Greenhouse Gas Emissions Reductions: Results from the EPPA Model Environmental Modeling \& Assessment August 2012, Volume 17, Issue 4, pp 325-336 Date: 27 Nov 2011. 